\title{
LE FUTUR DANS LES LANGUES ROMANES
}

Le Danois Rasmus Rask et l'Allemand Franz Bopp furent les deux fondateurs de la grammaire comparée du XIX siècle. Rask avait écrit en 1814 son étude sur l'origine de l'ancienne langue nordique, qui ne fut cependant publiée qu'en 1818. Et Bopp fit paraître en 1816 son ouvrage sur Ia flexion verbale du sanscrit. Très logiquement on fit faire alors à Rask un voyage aux Indes pour étudier cette langue indienne déjà mise à la mode par Schlegel.

En rentrant au Danemark, après une absence de sept ans, en 1824, Rask ne publia rien sur le sanscrit, mais une grammaire de l'espagnol, suivie d'une grammaire du frison (1825) et d'une grammaire de l'italien (1827). Ce qui l'intéressait avant tout, et ce à quoi il travailla toute sa vie, c'était en effet de rédiger une série de grammaires, construites de façon identique, de toutes les langues possibles, pour montrer ainsi les rapports qu'il y a entre elles. Il faisait de la grammaire comparée, sinon au sens tzaditionnel, du moins au sens propre du mot.

Dans cette grammaire espagnole de 1824, Rask discute l'origine du futur et du conditionnel dans les langues romanes, ou plutôt en espagnol. On connaissait déjà à cette époque les étymologies aujourd'hui universellement adoptées qui font remonter le futur espagnol cantará à cantare babet, et le conditionnel cantaría à cantare babebat. Mais Rask les rejette expressément, leur préférant cantare it et cantare ibat, formations qu'il compare lui-même aux périphrases françaises "il va chanter" et "il allait chanter". Il est intéressant de constater que le grand hispanisant danois de l'époque, J. L. Heiberg, auteur d'une thèse latine sur le théâtre de Calderón, accepte, dans une lettre à Rask, cette théorie curieuse en la qualifiant même d'astucieusè.

La théorie de Rask n'est d'ailleurs pas tout à fait impossible. Nous avons tous appris à l'école que les verbes de mouvement se construisent avec un supin: it cantatum, mais c'est là du latin classique, et le latin classique est 
dans ce cas, comme dans bien d'autres, une sorte de développement particulier qui s'écarte de l'évolution directe du latin ancien au latin vulgaire. L'emploi de l'infinitif final après un verbe de mouvement est en effet l'une des plus anciennes constructions infinitives. Chez Plaute, on trouve it cantare, et c'est la construction qu'on retrouve en latin vulgaire: venimus adorare eum (Matth. 2, 2), après la parenthése du latin clasique. Seulement, Rask ne tient pas compte du témoignage des textes qui prouvent que c'étaient bien habet cantare et habebat cantare qui devenaient en latin vulgaire des périphrases futurales, et non it cantare et ibat cantare.

Mais il y a plus grave. Rask commet la faute de donner une explication qui ne vaut que pour une seule langue romane, au lieu de tenir compte de l'ensemble des langues romanes, ce qui est vraiment paradoxal pour un fondateur de la grammaire comparée. Rask en est d'ailleurs conscient, car il remarque que sa théorie ne s'applique pas de façon aussi adéquate aux autres langues romanes. Pour ce qui est du conditionnel espagnol, on comprend qu'il ait été tenté de voir en ibat l'étymologie de la désinence -ia. Mais l'ancien français chantereit doit nécessairement avoir pour origine un $-\bar{e} a<b a b \bar{e} b a t$. Et le portugais a tout simplement conservé, avec sa tmèse, l'ancienne construction habere + infinitif: êle ver-te bá "il te verra".

Mais même en comparant tous les aboutissements romans de cantare babet, on n'aurait pas tenu compte de la totalité des langues romanes, car cantare habet n'est pas devenu le futur de toutes les langues romanes. Loin de là. La périphrase cantare babet a remplacé le futur latin dans les langues romanes occidentales (abstraction faite du rhéto-roman, qui a un futur formé de venio cantare) et en toscan, mais elle n'existe pas dans les langues romanes du Sud-Est. En Sardaigne et en Italie du Sud on emploie simplement le présent pour exprimer le futur, et en dalmate et en roumain on a eu recours à volo cantare.

La périphrase futurale cantare babet n'est donc pas commune à toutes les langues romanes. Ce qui leur est commun, c'est seulement la disparition du futur latin. A un certain moment, le latin vulgaire n'a pas eu de futur du tout. C'est l'état linguistique que représente le texte Peregrinatio Aetheriae, dans lequel il n'y a pas un seul futur. Cette disparition du futur, comment s'explique-t-elle ?

D'après Karl Vossler il s'agirait d'un phénomène propre à une langue vulgaire: "Aber der ganze Zeitbegriff des Futurums war schwach und ging in die Brüche. Er ist dem niederen Volk wohl kaum in einer Sprache sonderlich geläufig" (Geist und Kultur in der Sprache, 1925, p. 67 ss). Or, on a la preuve du contraire dans la renaissance du futur. Si la disparition 
du futur était l'état naturel de la langue, pourquoi certains dialectes du latin vulgaire se seraient-ils recréé un futur comme cantare babet?

Louis Hjelmslev, comme mâitre de conférences à l'université de Aarhus en 1933, consacra ses premiers cours aux rapports entre le système linguis. tique et le changement linguistique (publiés, en danois, en 1972: Sprogsystem og sprogforandring). Dans cette étude il propose dexpliquer tous les changements linguistiques à partir des systèmes, donc de rendre compte de la diachronie par la synchronie. Un système contient certaines tendances, ditil, qui, contenues pendant des périodes stables, se développent librement quand elles sont favorisées par certaines situations chaotiques de l'histoire.

Il y a certainement du vrai dans cette théorie, et l'on pourrait ainsi s'expliquer pourquoi les tendances de la langue de Plaute ne remontent définitivement à la surface que dans celle de Pétrone. Et si nous appliquons le principe au problème du futur roman, ce que Hjelmslev ne fait pas, nous pourrons dire que la disparition du futur est l'aboutissement d'une tendance du latin vulgaire qui aurait existé depuis Plaute. Or, encore une fois, et c'est là, me parait-il, un argument sérieux contre la théorie de Hjelmslev, on ne voit pas comment une tendance du système lat'n pourrait conduire à des résultats différents dans les langues romanes.

Le seul moyen de résoudre le problème de la disparition du futur latin est de trouver une explication systématique qui soit en même temps historique, et qui tienne compte du fait que la plupart des langues romanes se soient créé un nouveau futur pour le remplacer. Cette explication, on l'a trouvée il y a longtemps. Le futur latin est devenu inutilisable parce que, sous le coup de l'évolution phonétique, ses formes se confondaient, de façon intolérable, avec celles d'autres temps verbaux. Le futur de la première conjugaison cantabit entrait en collision avec le parfait cantavit, et le futur de la troisième conjugaison scribet avec le présent scribit. La langue latine a donc perdu son futur malgré elle, et non en vertu d'une tendance. Et c'est cela qui explique qu'on ait pu sentir le besoin de réintroduire le futur.

Le grand problème est de savoir pourquoi ce besoin s'est fait sentir dans certaines langues, et pas dans d'autres, et pourquoi, dans le premier cas, les résultats ont été différents. En gros, on peut dire que ces résultats divisent la Romania en quatre régions, comme nous l'avons dejà dit: 1. les langues romanes occidentales (moins le théto-roman) et le toscan: cantare babet $>$ fr. chantera, etc., 2. le rhéto-roman (c'est-à-dire, le romanche et l'engadinois, non le frioulan) : venit cantare $>$ romanche ven'el cantar, etc., 3. le dalmate et le roumain: vult cantare $>$ roumain va cîntà, etc., 4 . le sarde et l'italien méridional: emploi du présent pour le futur. 
On peut encore simplifier la situation en divisant le domaine roman en deux: l'Ouest et la Toscane, où s'est formé un véritable nouveau futur synthétique: it canterà, etc., 2. le Sud-Est (et le rhéto-roman), où l'on s'est contenté de périphrases ou de rien du tout. Nous nous occuperons d'abord de ce dernier domaine, pour mettre ainsi mieux en relief les difficultés du grand problème de savoir pourquoi a été créé le nouveau futur synthétique.

On peut dire que dans cette région du Sud-Est, qu'on sait conservatrice à bien d'autres égards, les langues s'en sont tenues à l'état du latin vulgaire et ont accepté la disparition du futur synthétique en le remplaçant tout s mplement par le présent. Cela vaut avant tout pour le sarde et pour l'italien méridional, où l'on n'a même pas eu recours à des périphrases pour remplacer le futur, se contentant du présent tout court. Ce sont là, en effet, les langues les plus conservatrices de la Romania.

En roumain, on a eu recours à la périphrase volo cantare $>$ voi cintá. Weigand a voulu expliquer ce développement par le substrat thrace, puisque la même périphrase existe en albanais. Mais Kr. Sandfeld a prouvé, dans sa thèse sur l'infinitif des langues balkaniques (1900) et dans sa Linguistique balkanique (1930), que la périphrase, ou plutôt les périphrases qui se sont succédé de façon parallèle dans toutes les langues balkaniques, vien. nent du neo-grec. C'est donc l'adstrat qui est à l'origine de cette construction futurale du roumain.

Sur le dalmate on est mal renseigné, car Bartoli a seulement pu trouver des traces discutables de volo cantare dans ce dialecte roman (Das Dalmatische, 1906, \& 534).

Enfin, en rhéto-roman, c'est sans doute sous l'influence du futur germanique er wird singen qu'on a fait du verbe venire un auxiliaire du futur: venit cantare > romanche ven'el cantar. Il n'y a là rien de surprenant puis. que le romanche est très fortement influencé par le germanique à tous les égards. C'est que, ici, au centre de la Suisse, le germanique et le roman vivent tellement côte à côte qu'il vaudrait mieux parler de constrat que d'adstrat.

Si l'emploi du présent pour le futur ou du futur périphrastique est relativement simple à comprendre, il en va tout autrement du futur synthétique des langues romanes de l'Ouest, lequel reste toujours une énigme. Estce qu'il s'agit là encore d'une influence externe, du substrat, du superstrat ou de l'adstrat? Pour le substrat et le superstrat, disons tout de suite que cela n'est possibile: cet énorme territoire n'a eu ni le même substrat, ni le même superstrat. Cependant on a tenté une explication par l'adstrat.

Einar Löfstedt a suggéré qu'il pourrait s'agir d'une influence grecque: "Zurückzuführen ist der ganze Gebrauch vor allem auf die Bedeutung des 
Müssens, Sollens, die dem mit Infinitiv verbundenen habere - zum Teil vielleicht unter Einfluss von griech. $\tilde{\varepsilon} \chi \omega$ nicht selten anhaftet (Zur Auflösung des Futurums und des Lokativs. Syntactica II, 1950, p. 63-73). Mais deux objections doivent faire écarter cette théorie: premièrement, le grec n'a justement pas eu recours au verbe $\check{\not} \chi \omega$ pour former le futur, mais au verbe $\Theta \varepsilon ́ \hat{\lambda} \omega$; deuxièmement une telle explication ne rendrait pas compte de la division de la Romania en ce qui concerne la formation du futur.

Il en est de même de l'influence chrétienne, qui, selon Coseriu (Sobre el futuro romance, Revista Brasileira de Filologia III, 1957, p. 3-18), serait a l'origine du nouveau futur cantare habet, périphrase à laquelle le christianisme aurait donné un sens éthique particulier. En effet cette périphrase se trouve d'abord et avant tout sous la plume du père de l'Eglise Tertullien, mais cet argument ne suffit pas. D'abord, on sait que les grands courants de la civilisation n'ont guère d'influence sur les langues. Ils en ont une, très forte parfois, sur le vocabulaire et le style, mais pas sur la grammaire proprement dite. Ensuite, et c'est là l'objection captale, on ne voit pas comment cette influence chrétienne pourrait justifier la dislocation de la Romania à cet égard. Est-ce que les différences religieuses entre l'Eglise grecque et l'Eglise romaine suffisent pour rendre compte de la distribution de vult cantare et de habet cantare? Et est-ce qu'il n'y a pas eu de christianisme en Sardaigne ou au sud de Rome ? Il est vrai que Carlo Levi a écrit un roman intitulé "Cristo si è fermato a Eboli" (1941).

Au lieu de telles explications externes, nous voudrions tenter une explication historique interne ou structurale. On a tendance, et Saussure luimême d'ailleurs, à identifier structure et synchronie, mais la structure joue aussi un rôle, et capital, dans la diachronie, qui, elle aussi, doit être structurale. Nous avons déjà donné une explication structurale de la disparition du futur synthétique latin. Pourrait-on aussi en donner une de la création du futur synthétique roman? Cela semble, en effet, possible.

Jusqu'ici nous n'avons guère parlé que du futur, et non du conditionnel. Or, on constate que toutes les langues romanes se sont créé un conditionnel mais c'est seulement là où le conditionnel est formé de cantare babebat qu'on a eu un futur formé de cantare babet. En sarde, debebam cantare a donné dio kantare. En italien méridional, on a un conditionnel formé du plus-que-parfait latin cantaverat $>$ kantara. En dalmate et en roumain la base du nouveau conditionnel est le futur antérieur cantaverit $>$ dalmate kanture, ancien roumain cîntare, roumain moderne ar cîntá. En théto-roman, c'est le plus-que-parfait du subjonctif cantavisset qui donne cantass.

L'italien au nord de Rome pose des problèmes particulièrement ardus, mais qui concernent plutôt le conditionnel que son rapport avec le futur. En 
toscan, le conditionnel est formé de cantare babuit $>$ canterebbe, au lieu de cantare habebat, mais le parallélisme avec le futur canterá reste le même. En lombard, on a un conditionnel canteresse, qui remonte probablement à cantare babuisset, donc toujours une formation de l'infinitif + babere. Il semble $y$ avoir une espèce de transition naturelle du nord au sud: cantavisset (rhéto-roman), cantare babuisset (lombard), cantare habuit (toscan), cantaverat (italien méridional).

Si nous avons donné une explication structurale de la création du futur synthétique cantare babet dans les langues romanes de l'Ouest, à savoir par son parallélisme avec le conditionnel formé de cantare habebat (babuit), nous nous trouvons ainsi devant une situation qui caractérise de façon typique toutes les explications structurales de ce genre. En effet, en expliquant une structure quelconque du système par une autre structure, on doit se poser la même question à propos de celle-ci. Et dans le cas présent: comment s'explique cette répartition des formes du conditionnel ?

Ce qui frappe tout de suite, c'est que le conditionnel remontant à cantaverat, cantaverit, cantavisset est un conditionnel mode, tandis que le conditionnel formé de cantare babebat (ou babuit) est en premier lieu un conditionnel temps, c'est-à-dire une transposition du futur au passé. Il semble donc qu'on puisse expliquer l'introduction de ce conditionnel temps par le style indirect qui est, dans une large mesure, une innovation des langues romanes, où il remplace la construction accusatif + infinitif. C'est ce que prouvent déjà les premiers exemples de la périphrase cantar $r e+$ habere, que l'on trouve chez Tertullien.

Dans Tertullien, on trouve 80 exemples de la périphrase, la plupart construits avec habebat, donc des conditionnels. Dans l'Ancien Testament, la Vulgate dit à propos de Daniel: civitatem exterminabit. En latin clas. sique, cela aurait été transposé en un accusatif + infinitif du style indirect: Daniel praedicavit civitatem esse exterminandam, donc avec un participe futur du passif. En remplaçant la construction infinitive par une complétive, il aurait fallu employer un futur antérieur du subjonctif du passif. Or, cette forme n'existe pas. Et c'est pourquoi Tertullien a recours à la périphrase: Daniel praedicavit quoniam civitas exterminari baberet.

Le conditionnel temps a donc été rendu nécessaire par le style indirect avant même que l'ancien futur ait disparu. Et il a pu ainsi former le modèle du nouveau futur. Mais, comme c'est toujours le cas de ces explications structurales, on peut à chaque étape reposer sa question: pourquoi le style indirect a-t-il seulement provoqué l'emploi du conditionnel temps à I'Ouest, et non au Sud-Est? 
On peut d'abord constater qu'il y a, en tout cas, une différence très frappante entre les styles indirects de ces deux grandes régions. Dans l'Ouest, toutes les propositions complétives sont introduites par que $(<$ quid $\leftarrow$ quod), au Sud-Est on a deux conjonctions spécialisées. En italien méridional, on emploie che (ou $m u$ ) après les verbes volitifs, ca ( $<$ quia) après les verbes déclaratifs. En roumain, les verbes volitifs sont suivis de să $(<s \bar{\imath})+$ le subjonctif, les verbes déclaratifs de că $(<q u i a)+$ l'indicatif. Cela veut dire que dans l'Ouest le conditionnel temps s'emploie après que là où le Sud-Ouest a ca.

Cela ne suffit cependant pas pour expliquer qu'il n'y a pas de conditionnel temps au Sud-Est. La raison en est plutôt qu'il n'y a pas, dans cette région, d'imparfait du subjonctif. Ni l'italien méridional ni le roumain n'ont d'imparfait du subjonctif. En Italie du Sud, le présent du subjonctif a disparu et a été remplacé par le plus-que-parfait du subjonctif ou tout simplement par l'indicatif. Et en roumain, le plus-que-parfait du subjonctif est devenu un plus-que-parfait de l'indicatif. Dans ces langues, il n'y a pas de concordance des temps au subjonctif. Par exemple en roumain, on fait suivre un imparfait de $s \breve{a}+$ le présent du subjonctif: Eram să vă spun "j'étais sur le point que je vous dise". C'est pourquoi on n'a pas non plus eu besoin de la concordance des temps à l'indicatif: Ştiam că de cealaltă parte voiu găsi casa podarului (Sandfeld et Hedvig Olsen, Syntaxe roumaine I, §326) “je savais que de l'autre côté je vais trouver la maison du batelier".

Mais pourquoi n'y a-t-il pas d'imparfait du subjonctif dans les langues du Sud-Est? Et c'est ainsi que les questions continuent à se poser par répercussion, quand on veut donner des explications structurales historiques.

KNUD TOGEBY

Université de Copenhague. 